\title{
Statistical comparison of quark mass matrices in the physical basis
}

\author{
S. Chaturvedi* \\ School of Physics. University of Hyderabad. \\ Hyderabad 500 046. INDIA. \\ V. Guptađ and G. Sánchez-Colón‡ \\ Departamento de Física Aplicada. \\ Centro de Investigación y de Estudios Avanzados del IPN. \\ Unidad Mérida. \\ A.P. 73, Cordemex. \\ Mérida, Yucatán, 97310. MEXICO. \\ S. Rajpoot $\$$ \\ Department of Physics $\&$ Astronomy. \\ California State University, Long Beach. \\ Long Beach, CA 90840. USA.
}

(Dated: September 1, 2018)

\begin{abstract}
Using the four best measured moduli of the flavor mixing matrix ( $\left.\left|V_{\mathrm{ud}}\right|,\left|V_{\mathrm{us}}\right|,\left|V_{\mathrm{cd}}\right|,\left|V_{\mathrm{cs}}\right|\right)$, the Jarlskog invariant $J(V)$, and the quark masses at $M_{Z}$ energy scale as experimental constraints, a statistical comparison of three different types of quark mass matrices in the physical basis is performed. The mass matrices in question are the Chaturvedi-Gupta-Sánchez-Colón (CGS), the Fritzsch and the Gupta-Rajpoot types. With nine parameters the best fits are obtained using a Gupta-Rajpoot type matrix while with seven parameters the best fits are obtained using the CGS type matrix. The stability of our analysis with respect to evolution of the quark masses is also presented.
\end{abstract}

PACS numbers: 13.30.Eg, 11.30.Er, 11.30.Hv, 12.60.-i

Keywords: mass matrix, mixing

\footnotetext{
*scsp@uohyd.ernet.in virendra@mda.cinvestav.mx †gsanchez@mda.cinvestav.mx $\sqrt[8]{\text { rajpoot@csulb.edu }}$
} 


\section{INTRODUCTION}

An important aspect of the standard model is the understanding of the structure of quark mass matrices that adequately describe the observed quark flavor mixing. The hope is that such studies will unravel the underlying symmetries responsible for the required texture in such mass matrices. Deviations form the symmetries will provide important constraints on possible new physics beyond the standard model.

In general, the $3 \times 3$ hermitian quark mass matrices $M_{\mathrm{u}}$ and $M_{\mathrm{d}}$ have nine real parameters each. These determine the six quark masses and the four independent real parameters in the quark mixing matrix $V$. Unfortunately, up to now one has no guiding principle for choosing $M_{\mathrm{u}}$ and $M_{\mathrm{d}}$ with fewer parameters. Recently [1, 2], constraints on three type of quark mass matrices were obtained using the experimentally determined values of the quark masses and measured properties of the mixing matrix $V$. In this paper we extend the analysis of Refs. [1, 2] to a different choice of mass matrices in the physical basis, namely, Fritzsch [3- 5], Gupta-Rajpoot [6, 7], and CGS [1, 2].

Fritzsch [3-5] suggested use of $M_{\mathrm{u}}$ and $M_{\mathrm{d}}$ with three textures (viz. $M_{11}=M_{22}=$ $\left.M_{13}=0\right)$. In this case, each quark mass matrix has five real parameters in general. The Gupta-Rajpoot [6, 7] mass matrix is similar to the Fritzsch type with one less texture, namely $M_{22} \neq 0$. Each Gupta-Rajpoot type of mass matrix has six real parameters in general. Lastly, we consider the CGS [1, 2] type matrix suggested recently. This has only two textures, $M_{11}=M_{22}=0$. For $M_{13}=0$ this reduces to the Fritzsch type matrix. CGS type matrix has the virtue that it will give $\mathrm{CP}$-violation in all the three bases (physical, $M_{\mathrm{u}}$ diagonal or $M_{\mathrm{d}}$ diagonal) because it can have $\operatorname{Im}\left(M_{12} M_{23} M_{13}^{*}\right) \neq 0$ [1]. Note that Fritzsch and Gupta-Rajpoot type matrices will give CP-violation only in the physical basis. In general, each CGS type of matrix has seven parameters. However, a unitary transformation relates both $M_{\mathrm{u}}$ and $M_{\mathrm{d}}$ of CGS type to, for example, $M_{\mathrm{u}}$ Fritzsch type and $M_{\mathrm{d}}$ CGS type.

Section III gives notation and basic formulas or expressions needed and the general procedure adopted for the analysis. Confrontation with experimental data for each type of matrices is presented in Secs. III, IV, and V, were we consider in the physical basis the Fritzsch, the Gupta-Rajpoot, and the CGS types of mass matrices, respectively. Results obtained for the various cases are compared and discussed in the final Sec. VI.

\section{NOTATION AND BASIC FORMULAS}

The $3 \times 3$ hermitian quark mass matrix $M_{\mathrm{q}}$ is diagonalized by $V_{\mathrm{q}}$ so that $M_{\mathrm{q}}=V_{\mathrm{q}}^{\dagger} \hat{M}_{\mathrm{q}} V_{\mathrm{q}}$, $\mathrm{q}=\mathrm{u}, \mathrm{d}$. The eigenvalues are denoted by $\left(\lambda_{\mathrm{u}}, \lambda_{\mathrm{c}}, \lambda_{\mathrm{t}}\right)$ and $\left(\lambda_{\mathrm{d}}, \lambda_{\mathrm{s}}, \lambda_{\mathrm{b}}\right)$ for the up and down quark mass matrices, respectively. Note that the eigenvalues are real but not necessarily positive. Each mass matrix can be expressed in terms of its projectors. Thus,

$$
M_{\mathrm{u}}=\sum_{\alpha=\mathrm{u}, \mathrm{c}, \mathrm{t}} \lambda_{\alpha} N_{\alpha} \quad \text { and } \quad M_{\mathrm{d}}=\sum_{j=\mathrm{d}, \mathrm{s}, \mathrm{b}} \lambda_{j} N_{j} .
$$

Since $V=V_{\mathrm{u}} V_{\mathrm{d}}^{\dagger}$, it follows that [8]

$$
\left|V_{\alpha j}\right|^{2}=\operatorname{Tr}\left[N_{\alpha} N_{j}\right]
$$

where 


$$
N_{\alpha}=\frac{\left(\lambda_{\beta}-M_{\mathrm{u}}\right)\left(\lambda_{\gamma}-M_{\mathrm{u}}\right)}{\left(\lambda_{\beta}-\lambda_{\alpha}\right)\left(\lambda_{\gamma}-\lambda_{\alpha}\right)}
$$

and

$$
N_{j}=\frac{\left(\lambda_{k}-M_{\mathrm{d}}\right)\left(\lambda_{l}-M_{\mathrm{d}}\right)}{\left(\lambda_{k}-\lambda_{j}\right)\left(\lambda_{l}-\lambda_{j}\right)},
$$

with $(\alpha, \beta, \gamma)$ and $(j, k, l)$ any permutation of $(\mathrm{u}, \mathrm{c}, \mathrm{t})$ and $(\mathrm{d}, \mathrm{s}, \mathrm{b})$, respectively. By unitarity only four of the nine $\left|V_{\alpha j}\right|^{2}$ are independent.

The Jarlskog invariant $J(V)$, which is a measure of CP-violation can be directly expressed in terms of $M_{\mathrm{u}}$ and $M_{\mathrm{d}}$ and their eigenvalues [9], thus

$$
\operatorname{Det}\left(\left[M_{\mathrm{u}}, M_{\mathrm{d}}\right]\right)=2 i D\left(\lambda_{\alpha}\right) D\left(\lambda_{j}\right) J(V),
$$

where $D\left(\lambda_{\alpha}\right)=\left(\lambda_{\mathrm{c}}-\lambda_{\mathrm{u}}\right)\left(\lambda_{\mathrm{t}}-\lambda_{\mathrm{u}}\right)\left(\lambda_{\mathrm{t}}-\lambda_{\mathrm{c}}\right)$ and $D\left(\lambda_{j}\right)=\left(\lambda_{\mathrm{s}}-\lambda_{\mathrm{d}}\right)\left(\lambda_{\mathrm{b}}-\lambda_{\mathrm{d}}\right)\left(\lambda_{\mathrm{b}}-\lambda_{\mathrm{s}}\right)$.

For a given choice of the mass matrices texture, most of the elements of the quark mass matrices can be expressed in terms of the mass eigenvalues (the free parameters) using the characteristic equations for $M_{\mathrm{u}}$ and $M_{\mathrm{d}}$ and others have to be considered as supplemental free parameters. We perform a $\chi^{2}$ analysis with a single $\chi^{2}$ function formed by eleven constraints [10] $\left|V_{\mathrm{ud}}^{\exp }\right|,\left|V_{\mathrm{us}}^{\exp }\right|,\left|V_{\mathrm{cd}}^{\exp }\right|,\left|V_{\mathrm{cs}}^{\exp }\right|, J^{\exp }(V)$, and $\lambda_{\mathrm{q}}^{\exp }\left(= \pm m_{\mathrm{q}}^{\exp }, \mathrm{q}=\mathrm{u}, \mathrm{c}, \mathrm{t}, \mathrm{d}, \mathrm{s}, \mathrm{b}\right)$, the eigenvalues of the quark mass matrices at a specified energy scale [11]. This function is

$$
\begin{aligned}
\chi^{2}= & \left(\frac{\left|V_{\mathrm{ud}}^{\exp }\right|-\left|V_{\mathrm{ud}}\right|}{\sigma_{\left|V_{\mathrm{ud}}\right|}}\right)^{2}+\left(\frac{\left|V_{\mathrm{us}}^{\exp }\right|-\left|V_{\mathrm{us}}\right|}{\sigma_{\left|V_{\mathrm{us}}\right|}}\right)^{2}+\left(\frac{\left|V_{\mathrm{cd}}^{\exp }\right|-\left|V_{\mathrm{cd}}\right|}{\sigma_{\left|V_{\mathrm{cd}}\right|}}\right)^{2}+\left(\frac{\left|V_{\mathrm{cs}} \exp \right|-\left|V_{\mathrm{cs}}\right|}{\sigma_{\left|V_{\mathrm{cs}}\right|}}\right)^{2}+ \\
& +\left(\frac{J^{\exp }(V)-J(V)}{\sigma_{J(V)}}\right)^{2}+\sum_{\mathrm{q}=\mathrm{u}, \mathrm{c}, \mathrm{t}, \mathrm{d}, \mathrm{s}, \mathrm{b}}\left(\frac{\lambda_{\mathrm{q}}^{\exp }-\lambda_{\mathrm{q}}}{\sigma_{\lambda_{\mathrm{q}}}}\right)^{2}
\end{aligned}
$$

This is an over constrained system of restrictions for the free parameters $\lambda_{\mathrm{q}}(\mathrm{q}=\mathrm{u}, \mathrm{c}, \mathrm{t}, \mathrm{d}, \mathrm{s}, \mathrm{b})$ and the supplemental free parameters for each texture if it is the case.

The numerical values [10, 11] of the eleven experimental constraints used (the six quark masses, the four moduli from the mixing matrix $\left(\left|V_{\mathrm{ud}}\right|\right.$, etc.), and $\left.J(V)\right)$ are given in Table \ for easy reference.

\section{FRITZSCH TYPE MASS MATRICES}

Fritzsch type mass matrices [3 -5] are given by the hermitian matrices

$$
M_{\mathrm{u}}=\left(\begin{array}{ccc}
0 & A & 0 \\
A^{*} & 0 & B \\
0 & B^{*} & C
\end{array}\right), \quad M_{\mathrm{d}}=\left(\begin{array}{ccc}
0 & A^{\prime} & 0 \\
A^{* *} & 0 & B^{\prime} \\
0 & B^{\prime *} & C^{\prime}
\end{array}\right) .
$$

Without lack of generality we can take $C$ and $C^{\prime}$ to be positive and $A$ and $B$ to be real and positive. Then, $M_{\mathrm{u}}$ and $M_{\mathrm{d}}$ have eight real parameters $A, B, C, C^{\prime},\left|A^{\prime}\right|,\left|B^{\prime}\right|$ and the phases $\phi_{A^{\prime}}$ and $\phi_{B^{\prime}}$. This type of of mass matrices can only be used in the physical basis because an off-diagonal matrix element is zero. 
From the characteristic polynomial of $M_{\mathrm{u}}$ and $M_{\mathrm{d}}$ the six parameters $A, B, C, C^{\prime},\left|A^{\prime}\right|$, and $\left|B^{\prime}\right|$ are expressed in terms of the eigenvalues

$$
\begin{gathered}
A=\left[-\frac{\lambda_{\mathrm{u}} \lambda_{\mathrm{c}} \lambda_{\mathrm{t}}}{\lambda_{\mathrm{u}}+\lambda_{\mathrm{c}}+\lambda_{\mathrm{t}}}\right]^{1 / 2}, \quad C=\lambda_{\mathrm{u}}+\lambda_{\mathrm{c}}+\lambda_{\mathrm{t}}, \\
B=\left[-\frac{\left(\lambda_{\mathrm{t}}+\lambda_{\mathrm{c}}\right)\left(\lambda_{\mathrm{t}}+\lambda_{\mathrm{u}}\right)\left(\lambda_{\mathrm{c}}+\lambda_{\mathrm{u}}\right)}{\lambda_{\mathrm{u}}+\lambda_{\mathrm{c}}+\lambda_{\mathrm{t}}}\right]^{1 / 2},
\end{gathered}
$$

and similarly for $M_{\mathrm{d}}$. The parameters $\left|A^{\prime}\right|,\left|B^{\prime}\right|$, and $C^{\prime}$ are obtained by replacing $(A, B, C)$ by $\left(\left|A^{\prime}\right|,\left|B^{\prime}\right|, C^{\prime}\right)$ and $\left(\lambda_{\mathrm{u}}, \lambda_{\mathrm{c}}, \lambda_{\mathrm{t}}\right)$ by $\left(\lambda_{\mathrm{d}}, \lambda_{\mathrm{s}}, \lambda_{\mathrm{b}}\right)$.

According to (2), the theoretical expressions for the magnitudes of the unitary quark mixing matrix elements in this case are given by

$$
\begin{aligned}
\left|V_{\alpha j}\right|^{2}= & {\left[\left(\lambda_{\alpha}-\lambda_{\beta}\right)\left(\lambda_{\alpha}-\lambda_{\gamma}\right)\left(\lambda_{j}-\lambda_{k}\right)\left(\lambda_{j}-\lambda_{l}\right)\right]^{-1} \times } \\
& \left\{\left(\lambda_{\beta} \lambda_{\gamma}+A^{2}+B^{2}\right)\left(\lambda_{k} \lambda_{l}+\left|A^{\prime}\right|^{2}+\left|B^{\prime}\right|^{2}\right)+\left(\lambda_{\beta} \lambda_{\gamma}+A^{2}\right)\left(\lambda_{k} \lambda_{l}+\left|A^{\prime}\right|^{2}\right)\right. \\
& +\left[\left(\lambda_{\alpha}+\lambda_{\beta}\right)\left(\lambda_{\alpha}+\lambda_{\gamma}\right)+B^{2}\right]\left[\left(\lambda_{j}+\lambda_{k}\right)\left(\lambda_{j}+\lambda_{l}\right)+\left|B^{\prime}\right|^{2}\right] \\
& +2\left(\lambda_{\beta}+\lambda_{\gamma}\right)\left(\lambda_{k}+\lambda_{l}\right) A\left|A^{\prime}\right| \cos \left(\phi_{A^{\prime}}\right) \\
& \left.+2 \lambda_{\alpha} \lambda_{j} B\left|B^{\prime}\right| \cos \left(\phi_{B^{\prime}}\right)+2 B A\left|B^{\prime}\right|\left|A^{\prime}\right| \cos \left(\phi_{A^{\prime}}+\phi_{B^{\prime}}\right)\right\},
\end{aligned}
$$

where $(\alpha, \beta, \gamma)$ is any permutation of $(\mathrm{u}, \mathrm{c}, \mathrm{t})$ and $(j, k, l)$ any permutation of $(\mathrm{d}, \mathrm{s}, \mathrm{b})$.

In this case, the theoretical expression for the Jarslkog invariant $J(V)$ given by Eq. (5) translates into

$$
\begin{aligned}
J(V)= & {\left[\left(\lambda_{\mathrm{t}}-\lambda_{\mathrm{c}}\right)\left(\lambda_{\mathrm{t}}-\lambda_{\mathrm{u}}\right)\left(\lambda_{\mathrm{c}}-\lambda_{\mathrm{u}}\right)\left(\lambda_{\mathrm{b}}-\lambda_{\mathrm{s}}\right)\left(\lambda_{\mathrm{b}}-\lambda_{\mathrm{d}}\right)\left(\lambda_{\mathrm{s}}-\lambda_{\mathrm{d}}\right)\right]^{-1} \times } \\
& \left\{\left[A\left|A^{\prime}\right| \sin \left(\phi_{A^{\prime}}\right)-B\left|B^{\prime}\right| \sin \left(\phi_{B^{\prime}}\right)\right]\right. \\
& \times\left[A^{2}\left|B^{\prime}\right|^{2}+B^{2}\left|A^{\prime}\right|^{2}-2 A B\left|A^{\prime}\right|\left|B^{\prime}\right| \cos \left(\phi_{A^{\prime}}+\phi_{B^{\prime}}\right)\right] \\
& \left.-A\left|A^{\prime}\right| \sin \left(\phi_{A^{\prime}}\right)\left[C^{2}\left|B^{\prime}\right|^{2}+B^{2} C^{\prime 2}-2 C B C^{\prime}\left|B^{\prime}\right| \cos \left(\phi_{B^{\prime}}\right)\right]\right\} .
\end{aligned}
$$

The mass matrices $M_{\mathrm{u}}$ and $M_{\mathrm{d}}$ in Eq. (7) do not have positive definite eigenvalues. Thus, $\lambda_{\mathrm{u}}^{2}=m_{\mathrm{u}}^{2}, \lambda_{\mathrm{d}}^{2}=m_{\mathrm{d}}^{2}$, etc., where $m_{\mathrm{u}}$ is the (positive) mass of the up quark, etc. Using the mass hierarchies $\left|\lambda_{\mathrm{u}}\right|<<\left|\lambda_{\mathrm{c}}\right|<<\left|\lambda_{\mathrm{t}}\right|$ for the up quark sector and $\left|\lambda_{\mathrm{d}}\right|<<\left|\lambda_{\mathrm{s}}\right|<<\left|\lambda_{\mathrm{b}}\right|$ for the down one and the characteristic equations it is possible to fix the relative phases between the eigenvalues and the quark masses [1],

$$
\left(\lambda_{\mathrm{u}}, \lambda_{\mathrm{c}}, \lambda_{\mathrm{t}}\right)=\left(m_{\mathrm{u}},-m_{\mathrm{c}}, m_{\mathrm{t}}\right) \quad \text { and } \quad\left(\lambda_{\mathrm{d}}, \lambda_{\mathrm{s}}, \lambda_{\mathrm{b}}\right)=\left(m_{\mathrm{d}},-m_{\mathrm{s}}, m_{\mathrm{b}}\right) .
$$

For this texture there are eight free parameters to be estimated, the six eigenvalues $\left(\lambda_{\mathrm{u}}, \lambda_{\mathrm{c}}, \lambda_{\mathrm{t}}\right)$ and $\left(\lambda_{\mathrm{d}}, \lambda_{\mathrm{s}}, \lambda_{\mathrm{b}}\right)$, and the two phases $\phi_{A^{\prime}}$ and $\phi_{B^{\prime}}$. The number of degrees of freedom is three (eleven constraints with eight free parameters). At the $M_{Z}$ energy scale the 
fitted values for the eigenvalues are $\lambda_{\mathrm{u}}=\left(1.66_{-0.36}^{+0.40}\right) \mathrm{MeV}, \lambda_{\mathrm{c}}=-(0.621 \pm 0.080) \mathrm{GeV}, \lambda_{\mathrm{t}}=$ $(172.4 \pm 3.0) \mathrm{GeV}, \lambda_{\mathrm{d}}=\left(2.29_{-0.49}^{+0.50}\right) \mathrm{MeV}, \lambda_{\mathrm{s}}=-\left(38.0_{-5.8}^{+4.3}\right) \mathrm{MeV}, \lambda_{\mathrm{b}}=(2.901 \pm 0.090) \mathrm{GeV}$, and the fitted values for the phases are $\phi_{A^{\prime}}=\left(-71_{-23}^{+19}\right)^{\circ}$ and $\phi_{B^{\prime}}=\left(-4_{-16}^{+18}\right)^{\circ}$. The total $\chi^{2} /($ dof $)=4.23 / 3=1.41$.

We observe that the fitted values obtained for the phases $\phi_{A^{\prime}}$ and $\phi_{B^{\prime}}$ are compatible with $-\pi / 2$ and 0 , respectively. The corresponding results obtained for this particular choice in this six free parameters fit (the six eigenvalues) and five degrees of freedom at the same $M_{Z}$ energy scale are: $\lambda_{\mathrm{u}}=\left(1.66_{-0.36}^{+0.40}\right) \mathrm{MeV}, \lambda_{\mathrm{c}}=-(0.622 \pm 0.079) \mathrm{GeV}, \lambda_{\mathrm{t}}=(172.4 \pm 3.0) \mathrm{GeV}$, $\lambda_{\mathrm{d}}=(1.98 \pm 0.22) \mathrm{MeV}, \lambda_{\mathrm{s}}=-\left(38.3_{-3.9}^{+4.0}\right) \mathrm{MeV}, \lambda_{\mathrm{b}}=(2.902 \pm 0.090) \mathrm{GeV}$. The total $\chi^{2} /($ dof $)=4.84 / 5=0.97$.

It is interesting to note that the last fit was obtained with only six real parameters because $M_{\mathrm{u}}$ is real and in $M_{\mathrm{d}}, B^{\prime}$ and $C^{\prime}$ are real and $A^{\prime}$ is pure imaginary.

\section{GUPTA-RAJPOOT TYPE MASS MATRICES}

For the three families of quarks the Gupta-Rajpoot mass matrices are of the form [6, 7]

$$
M_{\mathrm{u}}=\left(\begin{array}{ccc}
0 & A & 0 \\
A^{*} & |D| & B \\
0 & B^{*} & |C|
\end{array}\right), \quad M_{\mathrm{d}}=\left(\begin{array}{ccc}
0 & A^{\prime} & 0 \\
A^{\prime *} & \left|D^{\prime}\right| & B^{\prime} \\
0 & B^{\prime *} & \left|C^{\prime}\right|
\end{array}\right) .
$$

In their general form, these matrices have been extensively studied in the literature for both quarks mixing and lepton mixing [12]. Without lack of generality we can make $M_{\mathrm{u}}$ to be real and positive. Then, $M_{\mathrm{u}}$ and $M_{\mathrm{d}}$ have ten real parameters $A, B, C, D,\left|A^{\prime}\right|,\left|B^{\prime}\right|,\left|C^{\prime}\right|$, $\left|D^{\prime}\right|$, and the phases $\phi_{A^{\prime}}$ and $\phi_{B^{\prime}}$.

From the characteristic equations the parameters $A, B, C,\left|A^{\prime}\right|,\left|B^{\prime}\right|$, and $\left|C^{\prime}\right|$ are expressed in terms of the six eigenvalues and $D$. For $M_{\mathrm{u}}$,

$$
\begin{aligned}
& A=\left[-\frac{\lambda_{\mathrm{u}} \lambda_{\mathrm{c}} \lambda_{\mathrm{t}}}{\lambda_{\mathrm{u}}+\lambda_{\mathrm{c}}+\lambda_{\mathrm{t}}-D}\right]^{1 / 2}, \quad C=\lambda_{\mathrm{u}}+\lambda_{\mathrm{c}}+\lambda_{\mathrm{t}}-D, \\
B= & \left(\lambda_{\mathrm{u}}+\lambda_{\mathrm{c}}+\lambda_{\mathrm{t}}-D\right)^{-1 / 2} \times \\
& \left\{-\left(\lambda_{\mathrm{t}}+\lambda_{\mathrm{c}}\right)\left(\lambda_{\mathrm{t}}+\lambda_{\mathrm{u}}\right)\left(\lambda_{\mathrm{c}}+\lambda_{\mathrm{u}}\right)\right. \\
+ & {\left[\left(\lambda_{\mathrm{t}}+\lambda_{\mathrm{c}}\right)\left(\lambda_{\mathrm{t}}+\lambda_{\mathrm{u}}\right)+\left(\lambda_{\mathrm{t}}+\lambda_{\mathrm{u}}\right)\left(\lambda_{\mathrm{c}}+\lambda_{\mathrm{u}}\right)+\left(\lambda_{\mathrm{c}}+\lambda_{\mathrm{u}}\right)\left(\lambda_{\mathrm{t}}+\lambda_{\mathrm{c}}\right)\right] D } \\
& \left.-2\left(\lambda_{\mathrm{u}}+\lambda_{\mathrm{c}}+\lambda_{\mathrm{t}}\right) D^{2}+D^{3}\right\}^{1 / 2},
\end{aligned}
$$

and similarly for $M_{\mathrm{d}}$ where the parameters $\left|A^{\prime}\right|,\left|B^{\prime}\right|$, and $\left|C^{\prime}\right|$ are obtained by replacing $(A, B, C)$ by $\left(\left|A^{\prime}\right|,\left|B^{\prime}\right|,\left|C^{\prime}\right|\right),\left(\lambda_{\mathrm{u}}, \lambda_{\mathrm{c}}, \lambda_{\mathrm{t}}\right)$ by $\left(\lambda_{\mathrm{d}}, \lambda_{\mathrm{s}}, \lambda_{\mathrm{b}}\right)$, and $D$ by $\left|D^{\prime}\right|$.

According to Eq. (2), the theoretical expressions for the observable magnitudes of the unitary quark mixing matrix elements are given in this case by 


$$
\begin{aligned}
\left|V_{\alpha j}\right|^{2}= & {\left[\left(\lambda_{\alpha}-\lambda_{\beta}\right)\left(\lambda_{\alpha}-\lambda_{\gamma}\right)\left(\lambda_{j}-\lambda_{k}\right)\left(\lambda_{j}-\lambda_{l}\right)\right]^{-1} \times } \\
& \left\{\left(A^{2}+\lambda_{\beta} \lambda_{\gamma}\right)\left(\left|A^{\prime}\right|^{2}+\lambda_{k} \lambda_{l}\right)\right. \\
& +\left[B^{2}+\left(\lambda_{\alpha}+\lambda_{\beta}-D\right)\left(\lambda_{\alpha}+\lambda_{\gamma}-D\right)\right]\left[\left|B^{\prime}\right|^{2}+\left(\lambda_{j}+\lambda_{k}-\left|D^{\prime}\right|\right)\left(\lambda_{j}+\lambda_{l}-\left|D^{\prime}\right|\right)\right] \\
& +\left[A^{2}+B^{2}+\left(\lambda_{\beta}-D\right)\left(\lambda_{\gamma}-D\right)\right]\left[\left|A^{\prime}\right|^{2}+\left|B^{\prime}\right|^{2}+\left(\lambda_{k}-\left|D^{\prime}\right|\right)\left(\lambda_{l}-\left|D^{\prime}\right|\right)\right] \\
& +2\left(\lambda_{\beta}+\lambda_{\gamma}-D\right)\left(\lambda_{k}+\lambda_{l}-\left|D^{\prime}\right|\right) A\left|A^{\prime}\right| \cos \left(\phi_{A^{\prime}}\right) \\
& \left.+2 \lambda_{\alpha} \lambda_{j} B\left|B^{\prime}\right| \cos \left(\phi_{B^{\prime}}\right)+2 A B\left|A^{\prime}\right|\left|B^{\prime}\right| \cos \left(\phi_{A^{\prime}}+\phi_{B^{\prime}}\right)\right\}
\end{aligned}
$$

where $(\alpha, \beta, \gamma)$ is any permutation of $(\mathrm{u}, \mathrm{c}, \mathrm{t})$ and $(j, k, l)$ any permutation of $(\mathrm{d}, \mathrm{s}, \mathrm{b})$.

The theoretical expression for the observable Jarslkog invariant $J(V)$ for this case is obtained from Eq. (15),

$$
\begin{aligned}
J(V)= & {\left[\left(\lambda_{\mathrm{t}}-\lambda_{\mathrm{c}}\right)\left(\lambda_{\mathrm{t}}-\lambda_{\mathrm{u}}\right)\left(\lambda_{\mathrm{c}}-\lambda_{\mathrm{u}}\right)\left(\lambda_{\mathrm{b}}-\lambda_{\mathrm{s}}\right)\left(\lambda_{\mathrm{b}}-\lambda_{\mathrm{d}}\right)\left(\lambda_{\mathrm{s}}-\lambda_{\mathrm{d}}\right)\right]^{-1} \times } \\
& \left\{\left[A\left|A^{\prime}\right| \sin \left(\phi_{A^{\prime}}\right)-B\left|B^{\prime}\right| \sin \left(\phi_{B^{\prime}}\right)\right]\right. \\
& \times\left[A^{2}\left|B^{\prime}\right|^{2}+B^{2}\left|A^{\prime}\right|^{2}-2 A B\left|A^{\prime}\right|\left|B^{\prime}\right| \cos \left(\phi_{A^{\prime}}+\phi_{B^{\prime}}\right)\right] \\
& -A\left|A^{\prime}\right| \sin \left(\phi_{A^{\prime}}\right)\left[C^{2}\left|B^{\prime}\right|^{2}+B^{2} C^{\prime 2}-2 C B C^{\prime}\left|B^{\prime}\right| \cos \left(\phi_{B^{\prime}}\right)\right] \\
& +A B\left|D^{\prime}\right|\left[B\left|A^{\prime}\right| C^{\prime} \sin \left(\phi_{A^{\prime}}\right)+A\left|B^{\prime}\right| C^{\prime} \sin \left(\phi_{B^{\prime}}\right)-C\left|A^{\prime}\right|\left|B^{\prime}\right| \sin \left(\phi_{A^{\prime}}+\phi_{B^{\prime}}\right)\right] \\
& \left.+D\left|A^{\prime}\right|\left|B^{\prime}\right|\left[A C\left|B^{\prime}\right| \sin \left(\phi_{A^{\prime}}\right)+B C\left|A^{\prime}\right| \sin \left(\phi_{B^{\prime}}\right)-A B C^{\prime} \sin \left(\phi_{A^{\prime}}+\phi_{B^{\prime}}\right)\right]\right\} .
\end{aligned}
$$

To reduce the number of free parameters to nine we shall take $\left|D^{\prime}\right|=0$. As mentioned before, $\lambda_{\mathrm{u}}^{2}=m_{\mathrm{u}}^{2}, \lambda_{\mathrm{d}}^{2}=m_{\mathrm{d}}^{2}$, etc., and we need a solution with the mass hierarchies $\left|\lambda_{\mathrm{u}}\right|<<$ $\left|\lambda_{\mathrm{c}}\right|<<\left|\lambda_{\mathrm{t}}\right|$ for the up quark sector and $\left|\lambda_{\mathrm{d}}\right|<<\left|\lambda_{\mathrm{s}}\right|<<\left|\lambda_{\mathrm{b}}\right|$ for the down one. In this case the relative signs between the $\lambda$ 's and the quark masses can not be fixed a priori and all the possible combinations coming from the two signs in front of each of the quark masses were explored in the $\chi^{2}$ analysis. The result of this exploration is that the best fit for the Gupta-Rajpoot case is obtained for the identification, Eq. (11), of the relative signs between $\lambda$ 's and $m$ 's.

For this texture, the nine free parameters estimated were the six eigenvalues $\left(\lambda_{\mathrm{u}}, \lambda_{\mathrm{c}}, \lambda_{\mathrm{t}}\right)$ and $\left(\lambda_{\mathrm{d}}, \lambda_{\mathrm{s}}, \lambda_{\mathrm{b}}\right)$, the matrix element $D$, and the two phases $\phi_{A^{\prime}}$ and $\phi_{B^{\prime}}$. The number of degrees of freedom is then two. At the $M_{Z}$ energy scale the fitted values for the eigenvalues are $\lambda_{\mathrm{u}}=$ $\left(1.49_{-0.40}^{+0.43}\right) \mathrm{MeV}, \lambda_{\mathrm{c}}=-(0.610 \pm 0.083) \mathrm{GeV}, \lambda_{\mathrm{t}}=(172.5 \pm 3.0) \mathrm{GeV}, \lambda_{\mathrm{d}}=\left(3.19_{-0.80}^{+0.89}\right) \mathrm{MeV}$, $\lambda_{\mathrm{s}}=-\left(57_{-13}^{+14}\right) \mathrm{MeV}, \lambda_{\mathrm{b}}=\left(2.888_{-0.091}^{+0.089}\right) \mathrm{GeV}$, and the fitted values for the matrix element $D$ and the phases are $D=\left(2.6_{-2.0}^{+2.4}\right) \mathrm{GeV}, \phi_{A^{\prime}}=\left(-81_{-27}^{+28}\right)^{\circ}$ and $\phi_{B^{\prime}}=\left(-20_{-4}^{+12}\right)^{\circ}$, respectively. The total $\chi^{2} /($ dof $)=0.80 / 2=0.40$.

The fitted values obtained for the phases $\phi_{A^{\prime}}$ and $\phi_{B^{\prime}}$ are still compatible with $-\pi / 2$ and 0 , respectively. The corresponding results obtained for this particular choice in this seven free parameters fit (the six eigenvalues and $D$ ) and four degrees of freedom at the same $M_{Z}$ 
energy scale are: $\lambda_{\mathrm{u}}=\left(1.69_{-0.36}^{+0.40}\right) \mathrm{MeV}, \lambda_{\mathrm{c}}=-\left(0.591_{-0.081}^{+0.082}\right) \mathrm{GeV}, \lambda_{\mathrm{t}}=(172.5 \pm 3.0) \mathrm{GeV}$, $\lambda_{\mathrm{d}}=(2.92 \pm 0.69) \mathrm{MeV}, \lambda_{\mathrm{s}}=-(57 \pm 13) \mathrm{MeV}, \lambda_{\mathrm{b}}=(2.889 \pm 0.090) \mathrm{GeV}$, and $D=$ $\left(0.68_{-0.48}^{+0.52}\right) \mathrm{GeV}$. The total $\chi^{2} /($ dof $)=2.62 / 4=0.66$.

We note that the last fit was obtained with only seven free parameters since $M_{\mathrm{u}}$ has four real parameters while $M_{\mathrm{d}}$ is Fritzsch type with $A^{\prime}$ pure imaginary.

Another option to reduce the number of free parameters to nine is with $D=0$ and $\left|D^{\prime}\right| \neq 0$. All the possible combinations coming from the two signs in front of each of the quark masses were explored in the $\chi^{2}$ analysis and the best fit is obtained for the identification,

$$
\left(\lambda_{\mathrm{u}}, \lambda_{\mathrm{c}}, \lambda_{\mathrm{t}}\right)=\left(m_{\mathrm{u}},-m_{\mathrm{c}}, m_{\mathrm{t}}\right) \quad \text { and } \quad\left(\lambda_{\mathrm{d}}, \lambda_{\mathrm{s}}, \lambda_{\mathrm{b}}\right)=\left(-m_{\mathrm{d}}, m_{\mathrm{s}},-m_{\mathrm{b}}\right) .
$$

Now, the nine free parameters estimated were the six eigenvalues $\left(\lambda_{\mathrm{u}}, \lambda_{\mathrm{c}}, \lambda_{\mathrm{t}}\right)$ and $\left(\lambda_{\mathrm{d}}, \lambda_{\mathrm{s}}, \lambda_{\mathrm{b}}\right)$, the matrix element $\left|D^{\prime}\right|$, and the two phases $\phi_{A^{\prime}}$ and $\phi_{B^{\prime}}$. The number of degrees of freedom is two. At the $M_{Z}$ energy scale the fitted values for the eigenvalues are $\lambda_{\mathrm{u}}=$ $\left(1.60_{-0.37}^{+0.41}\right) \mathrm{MeV}, \lambda_{\mathrm{c}}=-(0.603 \pm 0.083) \mathrm{GeV}, \lambda_{\mathrm{t}}=(172.5 \pm 3.0) \mathrm{GeV}, \lambda_{\mathrm{d}}=-\left(3.13_{-0.79}^{+0.86}\right) \mathrm{MeV}$, $\lambda_{\mathrm{s}}=\left(55_{-13}^{+14}\right) \mathrm{MeV}, \lambda_{\mathrm{b}}=-(2.889 \pm 0.090) \mathrm{GeV}$, and the fitted values for the matrix element $\left|D^{\prime}\right|$ and the phases are $\left|D^{\prime}\right|=\left(32_{-23}^{+25}\right) \mathrm{MeV}, \phi_{A^{\prime}}=(101 \pm 23)^{\circ}$ and $\phi_{B^{\prime}}=\left(145_{-27}^{+41}\right)^{\circ}$, respectively. The total $\chi^{2} /($ dof $)=1.64 / 2=0.82$ compared to the 0.40 of the first choice..

In this case, the fitted values obtained for the phases $\phi_{A^{\prime}}$ and $\phi_{B^{\prime}}$ are compatible with $\pi / 2$ and $\pi$, respectively. The corresponding results obtained for this particular choice in this seven free parameters fit (the six eigenvalues and $\left|D^{\prime}\right|$ ) and two degrees of freedom at the same $M_{Z}$ energy scale are: $\lambda_{\mathrm{u}}=\left(1.70_{-0.36}^{+0.40}\right) \mathrm{MeV}, \lambda_{\mathrm{c}}=-(0.591 \pm 0.082) \mathrm{GeV}, \lambda_{\mathrm{t}}=(172.5 \pm$ $3.0) \mathrm{GeV}, \lambda_{\mathrm{d}}=(2.89 \pm 0.69) \mathrm{MeV}, \lambda_{\mathrm{s}}=-(56 \pm 13) \mathrm{MeV}, \lambda_{\mathrm{b}}=(2.889 \pm 0.090) \mathrm{GeV}$, and $\left|D^{\prime}\right|=(19 \pm 13) \mathrm{MeV}$. The total $\chi^{2} /($ dof $)=2.72 / 4=0.68$, very similar to the corresponding 0.66 of the first choice.

\section{CGS TYPE MASS MATRICES}

CGS-type hermitian mass matrix was considered first in Refs. [1, 2] and is given by

$$
M=\left(\begin{array}{ccc}
0 & a & d \\
a^{*} & 0 & b \\
d^{*} & b^{*} & c
\end{array}\right) .
$$

For $d=0$ this reduces to the Fritzsch type. This mass matrix has the virtue that it will give $\mathrm{CP}$-violation in all the three bases because $\operatorname{Im}\left(M_{12} M_{23} M_{13}^{*}\right) \neq 0$ [1]. To reduce the number of parameters to four we can take $a, b$, and $c$ to be real and with $d$ as pure imaginary.

In the up (down) quark diagonal basis $M=M_{\mathrm{d}}\left(M_{\mathrm{u}}\right)$ there are seven parameters, 4 in $M$ and 3 masses in the diagonal mass matrix.

In the physical basis with the CGS-type mass matrix it is enough to consider the case where $M_{\mathrm{u}}$ is Fritzsch-type while $M_{\mathrm{d}}$ is CGS-type [1, 2]

$$
M_{\mathrm{u}}=\left(\begin{array}{lll}
0 & a & 0 \\
a & 0 & b \\
0 & b & c
\end{array}\right), \quad M_{\mathrm{d}}=\left(\begin{array}{ccc}
0 & a^{\prime} & i\left|d^{\prime}\right| \\
a^{\prime} & 0 & b^{\prime} \\
-i\left|d^{\prime}\right| & b^{\prime} & c^{\prime}
\end{array}\right) .
$$

All the matrix elements are taken to be real and positive, so we have seven real parameters. This is a small (but important) variation of Eq. (77) above. 
There exists a unitary matrix $Y$ such that it can rotate $M_{u}$ and $M_{d}$ in Eq. (18) to mass matrices $M_{u}^{\prime}=Y^{\dagger} M_{u} Y$ and $M_{d}^{\prime}=Y^{\dagger} M_{d} Y$ which are CGS-type and Fritzsch-type respectively. Explicitly, the unitary matrix

$$
Y=\frac{1}{b}\left(\begin{array}{ccc}
\beta & \delta & 0 \\
\delta & \beta & 0 \\
0 & 0 & b
\end{array}\right)
$$

where $b^{2}=\beta^{2}+|\delta|^{2}$ and $\delta=i \eta_{\delta}|\delta|\left(\eta_{\delta}= \pm 1\right)$ is pure imaginary. This gives,

$$
M_{u}^{\prime}=\left(\begin{array}{ccc}
0 & a & \delta^{*} \\
a & 0 & \beta \\
\delta & \beta & c
\end{array}\right), \quad M_{d}^{\prime}=\left(\begin{array}{ccc}
0 & a^{\prime} & \delta^{\prime} \\
a^{\prime} & 0 & \beta^{\prime} \\
-\delta^{\prime} & \beta^{\prime} & c^{\prime}
\end{array}\right) .
$$

The conditions which make $Y$ a unitary matrix ensures $M_{u}^{\prime}$ and $M_{u}$ have the same eigenvalues. Further, in $M_{d}^{\prime}, b \beta^{\prime}=d^{\prime} \delta^{*}+\beta b^{\prime}$ and $b \delta^{\prime}=\beta d^{\prime}+b^{\prime} \delta^{*}$. Since $d^{\prime}$ and $\delta$ are pure imaginary so is $\delta^{\prime}$ while $\beta^{\prime}$ is real. Thus, both $M_{u}^{\prime}$ and $M_{d}^{\prime}$ are of the CGS-type. By requiring, $\delta^{\prime}=0$ we can make $M_{d}^{\prime}$ to be Fritzsch-type. Thus, it is sufficient to consider the case $M_{u}$ Fritzsch-type and $M_{d}$ CGS-type (see Eq. (18)) in this analysis.

From the characteristic polynomial of $M_{\mathrm{u}}$ and $M_{\mathrm{d}}$ we can solve for the matrix elements in terms of the corresponding eigenvalues and $\left|d^{\prime}\right|$,

$$
\begin{gathered}
a=\left[-\frac{\lambda_{\mathrm{u}} \lambda_{\mathrm{c}} \lambda_{\mathrm{t}}}{\lambda_{\mathrm{u}}+\lambda_{\mathrm{c}}+\lambda_{\mathrm{t}}}\right]^{1 / 2}, \quad c=\lambda_{\mathrm{u}}+\lambda_{\mathrm{c}}+\lambda_{\mathrm{t}}, \\
b=\left[-\frac{\left(\lambda_{\mathrm{t}}+\lambda_{\mathrm{c}}\right)\left(\lambda_{\mathrm{t}}+\lambda_{\mathrm{u}}\right)\left(\lambda_{\mathrm{c}}+\lambda_{\mathrm{u}}\right)}{\lambda_{\mathrm{u}}+\lambda_{\mathrm{c}}+\lambda_{\mathrm{t}}}\right]^{1 / 2},
\end{gathered}
$$

and

$$
\begin{aligned}
a^{\prime} & =\left[-\frac{\lambda_{\mathrm{d}} \lambda_{\mathrm{s}} \lambda_{\mathrm{b}}}{\lambda_{\mathrm{d}}+\lambda_{\mathrm{s}}+\lambda_{\mathrm{b}}}\right]^{1 / 2}, \quad c^{\prime}=\lambda_{\mathrm{d}}+\lambda_{\mathrm{s}}+\lambda_{\mathrm{b}}, \\
b^{\prime} & =\left[-\frac{\left(\lambda_{\mathrm{b}}+\lambda_{\mathrm{s}}\right)\left(\lambda_{\mathrm{b}}+\lambda_{\mathrm{d}}\right)\left(\lambda_{\mathrm{s}}+\lambda_{\mathrm{d}}\right)}{\lambda_{\mathrm{d}}+\lambda_{\mathrm{s}}+\lambda_{\mathrm{b}}}-\left|d^{\prime}\right|^{2}\right]^{1 / 2} .
\end{aligned}
$$

From Eq. (2), the theoretical expressions for the observables the magnitudes of the unitary quark mixing matrix elements in this case are

$$
\begin{aligned}
\left|V_{\alpha j}\right|^{2}= & {\left[\left(\lambda_{\beta}-\lambda_{\alpha}\right)\left(\lambda_{\gamma}-\lambda_{\alpha}\right)\left(\lambda_{k}-\lambda_{j}\right)\left(\lambda_{l}-\lambda_{j}\right)\right]^{-1} \times } \\
& {\left[\left(a b^{\prime}+b a^{\prime}\right)^{2}+2\left(a^{2} a^{\prime 2}+b^{2} b^{\prime 2}\right)+\left(a^{2}+b^{2}\right)\left|d^{\prime}\right|^{2}\right.} \\
& +2 a a^{\prime}\left(\lambda_{\beta}+\lambda_{\gamma}\right)\left(\lambda_{k}+\lambda_{l}\right)+\lambda_{\beta} \lambda_{\gamma} \lambda_{k} \lambda_{l} \\
& +\lambda_{k} \lambda_{l}\left(a^{2}+b^{2}+\lambda_{\alpha}^{2}\right)+\lambda_{\beta} \lambda_{\gamma}\left(a^{\prime 2}+b^{\prime 2}+\left|d^{\prime}\right|^{2}+\lambda_{j}^{2}\right) \\
& \left.+c \lambda_{\alpha}\left(b^{\prime 2}+\left|d^{\prime}\right|^{2}\right)+c^{\prime} \lambda_{j} b^{2}+\lambda_{\alpha} \lambda_{j}\left(c c^{\prime}+2 b b^{\prime}\right)\right],
\end{aligned}
$$

where $(\alpha, \beta, \gamma)$ is any permutation of $(\mathrm{u}, \mathrm{c}, \mathrm{t})$ and $(j, k, l)$ any permutation of $(\mathrm{d}, \mathrm{s}, \mathrm{b})$.

The theoretical expression for the Jarslkog invariant $J(V)$ given by Eq. (5) translates into, 


$$
J(V)=-\frac{b\left|d^{\prime}\right|\left[\left(a^{\prime} b-a b^{\prime}\right)\left(b c^{\prime}-b^{\prime} c\right)+a c\left|d^{\prime}\right|^{2}\right]}{\left(\lambda_{\mathrm{b}}-\lambda_{\mathrm{s}}\right)\left(\lambda_{\mathrm{b}}-\lambda_{\mathrm{d}}\right)\left(\lambda_{\mathrm{s}}-\lambda_{\mathrm{d}}\right)\left(\lambda_{\mathrm{t}}-\lambda_{\mathrm{c}}\right)\left(\lambda_{\mathrm{t}}-\lambda_{\mathrm{u}}\right)\left(\lambda_{\mathrm{c}}-\lambda_{\mathrm{u}}\right)} .
$$

As in the Fritzsch case it is possible to fix the relative signs between the eigenvalues and the quark masses using the quark mass hierarchies and the characteristic equations, Eqs. (21) and (22), again in this case are valid the identifications of Eq. (11).

The seven free parameters to be estimated are the six eigenvalues $\left(\lambda_{\mathrm{u}}, \lambda_{\mathrm{c}}, \lambda_{\mathrm{t}}\right)$ and $\left(\lambda_{\mathrm{d}}, \lambda_{\mathrm{s}}, \lambda_{\mathrm{b}}\right)$, and the matrix element $\left|d^{\prime}\right|$. The number of degrees of freedom is four. At the $M_{Z}$ energy scale the fitted values for these parameters are: $\lambda_{\mathrm{u}}=\left(1.30_{-0.43}^{+0.49}\right) \mathrm{MeV}, \lambda_{\mathrm{c}}=$ $-(0.643 \pm 0.081) \mathrm{GeV}, \lambda_{\mathrm{t}}=(172.4 \pm 3.0) \mathrm{GeV}, \lambda_{\mathrm{d}}=\left(2.79_{-0.38}^{+0.39}\right) \mathrm{MeV}, \lambda_{\mathrm{s}}=-(35.7 \pm 4.6) \mathrm{MeV}$, $\lambda_{\mathrm{b}}=(2.899 \pm 0.090) \mathrm{GeV}$, and $\left|d^{\prime}\right|=\left(8.3_{-1.1}^{+1.5}\right) \mathrm{MeV}$. The total $\chi^{2} /($ dof $)=1.89 / 4=0.47$.

This is a seven free parameters fit with $M_{\mathrm{u}}$ being Fritzsch type while $M_{\mathrm{d}}$ is CGS type with $a^{\prime}$ and $b^{\prime}$ real, and $d^{\prime}$ pure imaginary.

\section{CONCLUSIONS AND DISCUSSION OF RESULTS}

The results for the fits for all quark masses and matrix elements at the energy scale of $M_{Z}$ are summarized in column $M_{Z}$ of Table II. As can be seen, with 9 parameters the best fits are obtained using a Gupta-Rajpoot type matrix, as expected. However, fixing $\phi_{A^{\prime}}=-\pi / 2$ and $\phi_{B^{\prime}}=0$ (as suggested by the free fits) in the Fritzsch case, reducing the number of parameters to only 6 , gives a comparable $\chi^{2} /$ dof than fixing $\phi_{A^{\prime}}=-\pi / 2$ and $\phi_{B^{\prime}}=0$ (also suggested by the free fits and reducing the number of parameters to 7 ) in the Gupta-Rajpoot case. With 7 parameters the best ratio $\chi^{2} /$ dof is obtained using a CGS type matrix.

The stability of this type of analysis with respect to evolution of the quark masses is important, in column $2 \mathrm{GeV}$ of Table $\amalg$ we summarize the results for the different cases at $2 \mathrm{GeV}$ scale. As can be seen, the results for $\chi^{2} /$ dof are very similar to results at $M_{Z}$ scale.

A simple way to understand this is to note that if all quark masses are scaled by a common factor then the algebraic expressions for the dimensionless numbers $J(V)$ and the moduli $\left|V_{\alpha j}\right|(\alpha=\mathrm{u}, \mathrm{c}, \mathrm{j}=\mathrm{d}, \mathrm{s})$ will be unaffected. As can be seen from Table I the ratio $m_{\mathrm{q}}(2 \mathrm{GeV}) / m_{\mathrm{q}}\left(M_{Z}\right)=1.71-1.74$, for $\mathrm{q}=\mathrm{u}, \mathrm{d}, \mathrm{s}, \mathrm{c}, \mathrm{b}$, while it is 1.85 for $\mathrm{q}=\mathrm{t}$.

Finally, column PDG of Table II gives the results for the different cases for quark masses at different scales (the PDG convention) given in column PDG of Table I. Again, the results are similar and choice of CGS mass matrices is favored. Note that masses of the heavier quarks (notable $m_{\mathrm{t}}$ ) are very different. This would suggest that the role of the small masses (since they evolve slowly) is possibly more important.

In conclusion, we advocate the use of mass matrices which can be used in all the three bases.

\section{Acknowledgments}

V. Gupta and G. Sánchez-Colón would like to thank CONACyT (México) for partial support. The work of S. Rajpoot was supported by DOE Grant \#: DE-FG02-10ER41693.

[1] S. Chaturvedi, V. Gupta, and G. Sánchez-Colón, Int. J. of Mod. Phys. A 23 (2008) 1729. 
[2] V. Gupta, G. Sánchez-Colón, and S. Chaturvedi, AIP Conf. Proc. 1116 (2009) 101.

[3] H. Fritzsch, Phys. Lett. B 70 (1977) 436.

[4] H. Fritzsch, Phys. Lett. B 73 (1978) 317.

[5] H. Fritzsch, Phys. Lett. B 166 (1986) 423.

[6] S. N. Gupta and J. M. Johnson, Phys. Rev. D 44 (1991) 2110.

[7] S. Rajpoot, Mod. Phys. Lett. A 7 (1992) 309.

[8] C. Jarlskog, Phys. Rev. D 36 (1987) 2128.

[9] C. Jarlskog, Phys. Rev. Lett. 55 (1985) 1039.

[10] W. -M. Yao et al. (Particle Data Group), J. Phys. G: Nucl. Part. Phys. 33 (2006) 1.

[11] Z. Z. Xing, H. Zhang, and S. Zhou, Phys. Rev. D 77 (2008) 113016.

[12] S. Rajpoot, arXiv:hep-ph/0703185. This paper gives an extensive list of references to the study of the Gupta-Rajpoot scheme in its full generality. 
TABLE I: Quark masses at various energy scales. Those in columns 2 and 3 are the evolved masses taken from Ref. [11]. Column 4 gives masses given in Ref. [10]: $m_{\mathrm{u}}, m_{\mathrm{d}}$ and $m_{\mathrm{s}}$ are at $2 \mathrm{GeV}, m_{\mathrm{c}}$ at $m_{\mathrm{c}}, m_{\mathrm{b}}$ at $m_{\mathrm{b}}$ and $m_{\mathrm{t}}$ by direct observation. Note that $\mathrm{u}, \mathrm{d}$, and s masses are in MeV while those of c, b, and t are in GeV. The experimentally observed values [10] of the four best measured magnitudes of the quark mixing matrix elements and the Jarlskog invariant are displayed in the second part of the table.

\begin{tabular}{|c|c|c|c|}
\hline \multirow[t]{2}{*}{ Quark mass } & \multicolumn{3}{|c|}{ Scale } \\
\hline & $2 \mathrm{GeV}$ & $M_{Z}=91.1876 \mathrm{GeV}$ & PDG \\
\hline$m_{\mathrm{u}}$ & $2.2_{-0.7}^{+0.8}$ & $1.28_{-0.43}^{+0.50}$ & $2.25 \pm 0.75$ \\
\hline$m_{\mathrm{d}}$ & $5.0 \pm 2.0$ & $2.91_{-1.20}^{+1.24}$ & $5.0 \pm 2.0$ \\
\hline$m_{\mathrm{s}}$ & $95 \pm 25$ & $55_{-15}^{+16}$ & $95 \pm 25$ \\
\hline$m_{\mathrm{c}}$ & $1.07 \pm 0.12$ & $0.624 \pm 0.083$ & $1.25 \pm 0.09$ \\
\hline$m_{\mathrm{b}}$ & $5.04_{-0.15}^{+0.16}$ & $2.89 \pm 0.09$ & $4.20 \pm 0.07$ \\
\hline$m_{\mathrm{t}}$ & $318.9_{-12.3}^{+13.1}$ & $172.5 \pm 3.0$ & $174.2 \pm 3.3$ \\
\hline Observable & & Exp. value & \\
\hline$\left|V_{\mathrm{ud}}\right|$ & & $0.97383 \pm 0.00024$ & \\
\hline$\left|V_{\mathrm{us}}\right|$ & & $0.2272 \pm 0.0010$ & \\
\hline$\left|V_{\mathrm{cd}}\right|$ & & $0.2271 \pm 0.0010$ & \\
\hline$\left|V_{\mathrm{CS}}\right|$ & & $0.97296 \pm 0.00024$ & \\
\hline$J(V)$ & & $(3.08 \pm 0.18) \times 10^{-5}$ & \\
\hline
\end{tabular}

TABLE II: Fits for all quark masses and matrix elements at energy scales of $M_{Z}, 2 \mathrm{GeV}$, and at the PDG energy scales convention.

\begin{tabular}{c|c|c|c|c|c}
\hline Type of & Physical & Number of & \multicolumn{3}{|c}{$\chi^{2} /$ dof $)$} \\
\cline { 4 - 6 } mass matrix & Basis & param. & $M_{Z}$ & $2 \mathrm{GeV}$ & PDG \\
\hline Fritzsch & $\phi_{A^{\prime}}$ and $\phi_{B^{\prime}}$ free. & 8 & $4.23 / 3=1.41$ & $4.80 / 3=1.60$ & $3.32 / 3=1.11$ \\
& $\phi_{A^{\prime}}=-\pi / 2, \phi_{B^{\prime}}=0$. & 6 & $4.84 / 5=0.97$ & $5.49 / 5=1.10$ & $4.27 / 5=0.85$ \\
\hline Gupta-Rajpoot & $\phi_{A^{\prime}}$ and $\phi_{B^{\prime}}$ free. & 9 & $0.80 / 2=0.40$ & $0.87 / 2=0.44$ & $0.78 / 2=0.39$ \\
$\left|D^{\prime}\right|=0, D \neq 0$. & $\phi_{A^{\prime}}=-\pi / 2, \phi_{B^{\prime}}=0$. & 7 & $2.62 / 4=0.66$ & $2.76 / 4=0.69$ & $3.77 / 4=0.94$ \\
\hline Gupta-Rajpoot & $\phi_{A^{\prime}}$ and $\phi_{B^{\prime}}$ free. & 9 & $1.64 / 2=0.82$ & $1.77 / 2=0.89$ & $1.71 / 2=0.86$ \\
$\left|D^{\prime}\right| \neq 0, D=0$. & $\phi_{A^{\prime}}=\pi / 2, \phi_{B^{\prime}}=\pi$. & 7 & $2.72 / 4=0.68$ & $2.86 / 4=0.72$ & $3.83 / 4=0.96$ \\
\hline$M_{\mathrm{u}}$ Fritzsch-type & & 7 & $1.89 / 4=0.47$ & $2.47 / 4=0.62$ & $0.80 / 4=0.20$ \\
and $M_{\mathrm{d}}$ CGS-type. & & & & & \\
\hline
\end{tabular}

